\title{
C-terminal truncation of Pik3r1 in mice models human lipodystrophic insulin resistance uncoupled from dyslipidemia
}

\section{Correspondence to:}

Dr. Robert K. Semple, University of Cambridge Metabolic Research Laboratories, Institute of Metabolic Science, Box 289, Addenbrooke's Hospital, Cambridge CB2 0QQ, UK. Tel: +44 1223769 035/ Email: rks16@cam.ac.uk (LEAD CONTACT)

Conflict of Interest Statement: The authors have declared that no conflict of interest exists. 


\section{Summary}

3 Heterodimeric class IA phosphatidylinositol-3-kinases ( $\mathrm{PI} 3 \mathrm{~K})$ transduce signals from

4 many receptor tyrosine kinases including the insulin receptor. PI3K recruitment to

5 phosphotyrosines is mediated by Pik3r1 gene products including the most intensely

6 studied $\mathrm{PI} 3 \mathrm{~K}$ regulatory subunit, $\mathrm{p} 85 \alpha$, which also binds and regulates the $\mathrm{PIP}_{3}$

7 phosphatase Pten, and the lipogenic transcription factor Xbp1. Mutations in human

8 PIK3R1 cause SHORT syndrome, featuring lipodystrophy and severe insulin

9 resistance which, uniquely, are uncoupled from fatty liver and dyslipidemia. We

10 describe a novel mouse model of SHORT syndrome made by knock in of the Pik3r1

11 Y657X mutation. Homozygous embryos die at E11.5, while heterozygous mice

12 exhibit pre-and postnatal growth impairment with diminished placental vascularity.

13 Adipose tissue accretion on high fat feeding was reduced, however adipocyte size

14 was unchanged and preadipocyte differentiation ex vivo unimpaired. Despite severe

15 insulin resistance, heterozygous mice were hypolipidemic, and plasma adiponectin,

16 liver weight, cholesterol, glycogen and triglyceride content were unchanged. Mild

17 downregulation of lipogenic Srebp1, Srebp2 and Chrebp transcriptional activity but

18 no suppression of $X b p 1$ target genes was seen after fasting. These findings give

19 new insights into the developmental role of Pik3r1, and establish a model of

20 lipodystrophic insulin resistance dissociated from dyslipidemia as seen in SHORT

21 syndrome. 


\section{INTRODUCTION}

3 phosphatidylinositol-4,5-bisphosphate to produce phosphatidylinositol-3,4,5-

4 trisphosphate, or $\mathrm{PIP}_{3}$. They are composed of a heterodimer of a p110 catalytic

5 subunit (either $\mathrm{p} 110 \alpha, \beta$ or $\delta$, encoded in humans by PIK3CA, PIK3CB and PIK3CD respectively) tightly bound to a regulatory subunit, three of which - p85a,p55 $\alpha$ or

$7 \quad \mathrm{p} 50 \alpha-$ are encoded by the PIK3R1 gene. Each of these PIK3R1 gene products is able to bind any of the three catalytic subunits with no discernible selectivity, stabilising them and mediating recruitment of $\mathrm{PI} 3 \mathrm{~K}$ holoenzymes to activated receptor tyrosine kinases or their substrates through binding of one or both $\mathrm{SH} 2$ domains to phosphotyrosines within YMXM motifs (1, 2).

The critical importance of Class $1 \mathrm{~A} \mathrm{PI3K}$ in human physiology is manifest in

13 the convincing association of activating mutations in catalytic subunits with disease:

14 activating mutations in PIK3CA, encoding $\mathrm{p} 110 \alpha$, occur at high frequency both in

15 cancers (3), and in a wide range of asymmetric forms of overgrowth, where 16 mutations occur postzygotically and are in a mosaic distribution (4). Genetic and

17 pharmacological studies have moreover established that $p 110 \alpha$ is critical in 18 transducing the metabolic actions of insulin $(5,6)$, however it is also coupled to a 19 wide range of other receptor tyrosine kinases. $\mathrm{p} 110 \delta$ is predominantly important in lymphocytes, and activating mutations cause autosomal dominant immunodeficiency

21 (7), while the role of the ubiquitous $p 110 \beta$ has been less precisely defined (8). yielded a more complex picture. Selective deficiency of $p 85 \alpha$ in mice or humans leads to immunodeficiency $(9,10)$ and enhanced insulin sensitivity $(11)$, with the latter also seen in mice with heterozygous p85a deletion (12) or deletion of both 
1 mortality in mice with liver necrosis (14), and when the $p 85 \beta$ regulatory subunit,

2 encoded by Pik3r2, is also knocked out, embryos die around E12.5 with evidence of

3 failure of turning (15). Collectively these findings demonstrate that Pik3r1 gene

4 products and $p 85 \beta$ have some redundant functions. The enhanced insulin sensitivity

5 seen in p85 $\alpha$ deficiency has been argued to be due to the presence of excess free

$6 \mathrm{p} 85 \alpha$ subunits in the wild-type state which can compete with PI3K heterodimers at

7 phosphotyrosines (16), although there is some evidence to counter this notion, and

8 other possibilities such as alterations in $\mathrm{PIP}_{3}$ phosphatase activity have been

9 advanced (17).

$10 \quad$ PIK3R1 gene products have also been suggested to have signaling roles

11 beyond stabilising PI3K and conferring recruitability to phosphorylated YMXM motifs.

12 Pertinent to metabolic homeostasis, for example, is the ability of $p 85 \alpha$ in mice to bind

13 the transcription factor $\mathrm{Xbp} 1$ and to traffic it to the nucleus, where it regulates

14 transcription of effector genes of the unfolded protein response $(18,19)$, as well as

15 lipogenic genes (20).

16 In the face of this complexity, the discovery that missense or nonsense

17 mutations in the C-terminal $\mathrm{SH} 2$ domain of PIK3R1, which affect all three protein

18 products of the gene, produce SHORT syndrome (21-23), was of great interest.

19 SHORT syndrome is named according to visible dysmorphic features (short stature,

20 hyperextensibility of joints and/or hernia, ocular depression, Rieger anomaly of the

21 iris, and teething delay), however it is the associated metabolic disorders that are

22 potentially more informative about mechanisms of pandemic metabolic disease.

23 Most patients with SHORT syndrome show insulin resistance that is often very

24 severe, and partial lipodystrophy is also common (24). Highly unusually,

25 lipodystrophy and severe insulin resistance are uncoupled from fatty liver and

26 metabolic dyslipidaemia in this setting, for reasons that are not clear, and

27 adiponectin concentrations in the plasma are preserved, unlike in pandemic insulin 
1 resistance (25). Affected women are also commonly anovulatory with severely

2 elevated testosterone concentrations in the blood (25).

Recently, mice heterozygous for the commonest SHORT syndromeassociated PIK3R1 mutation, R649W, were reported to exhibit features of SHORT syndrome including reduced linear growth, partial lipodystrophy, and systemic insulin resistance (26). We now report a novel murine model of SHORT syndrome created

7 by knock in of a different pathogenic human allele, Y657X, that severely truncates

8 the $\mathrm{C}$ terminal $\mathrm{SH} 2$ domain of all PIK3R1 gene products. We extend previous

9 phenotyping to demonstrate the consequences for development of the Pik3r1

10 mutation, and, importantly, we demonstrate that this novel SHORT syndrome model

11 reproduces the unexplained uncoupling of metabolic dyslipidemia from

12 lipodystrophic insulin resistance seen in humans, although the most widely studied

13 lipogenic transcriptional programmes are only mildly perturbed.

\section{RESULTS}

16 Growth and development of Pik3r1 Y657X knockin mice

17 The truncating Pik3r1 Y657X mutation, previously associated with

18 normolipidemic severe insulin resistance in SHORT syndrome (25), was knocked

19 into murine embryonic stem cells by homologous recombination-based gene

20 targeting, and these cells were used to generate founder heterozygous knock-in

21 mice (Supplemental Figure S1). Immunoblotting of insulin-responsive liver,

22 skeletal muscle and adipose tissue confirmed the presence of a truncated p85 $\alpha$

23 gene product, which in general was more highly expressed than full length, wild type

24 protein in the same tissues, most likely due to loss of a previously described C-

25 terminal ubiquitylation motif (27) No change in expression was seen of the p85 $\beta$

26 regulatory subunit of $\mathrm{PI} 3 \mathrm{~K}$, while expression of the $\mathrm{p} 110 \alpha$ catalytic subunit was

27 reduced in white adipose tissue, and expression of the $p 110 \beta$ catalytic subunit was 
1 reduced in both liver and white adipose tissue. $p 110 \beta$ expression very low in skeletal

2 muscle in both wild-type and mutant animals (Supplemental Figure S1).

Homozygosity for the Pik3r1 Y657X allele was lethal in utero, with no

4 homozygous embryos identified beyond E11.5. At E11.5 homozygous embryos

5 were smaller, with poorly developed limb buds and reduced eye pigmentation

6 (Figure 1A), while heterozygous embryos were smaller from E15.5 onwards

7 (Supplemental Figure S2). No difference in mass of wild type and heterozygous

8 placentas examined from wild type dams was seen, however vascularisation of the

9 placental exchange region was severely compromised, with around a $40 \%$ reduction

10 in vessel density, volume and length at E15.5 (Supplemental Figure S3), as

11 reported previously for placentas heterozygous for a kinase dead $p 110 \alpha$ catalytic

12 subunit (28). In contrast to that model, however, the size of the placental exchange

13 region (both volume and surface area) and the thickness of the diffusion barrier were

14 normal (Supplemental Figure S3).

Pik3r1WT/Y657X mice were born at expected Mendelian frequency, however they showed impaired linear growth on a chow diet, with body length of males $6 \%$ less

17 than wild-type littermates at 18 weeks old (Figures 1B,C), and bodyweight $17 \%$ less

18 (Figure 1D). Overall body composition assessed by TD-NMR showed no difference

19 between heterozygous males and controls (Figure 1E), and, in keeping with this, no

20 difference was found in epididymal or inguinal white adipose depot nor interscapular

21 brown adipose depot weights when the reduced bodyweight of the male heterozygous mice was taken into account (Figure 1F-H). Plasma leptin concentrations were similar between male Pik3r1 ${ }^{\text {WTY } 657 X}$ and wild-type mice $(3.3 \pm 1.2$

$24 v s 2.6 \pm 0.3 \mu \mathrm{g} / \mathrm{L}$ in the fasting state $(\mathrm{n}=9,10$; not significant), and $10.3 \pm 2.2 \mathrm{vs}$ $15.9 \pm 3.9 \mu \mathrm{g} / \mathrm{L}$ on ad libitum feeding ( $\mathrm{n}=13,13$; not significant)). Liver weights were

26 also indistinguishable (Figure 1I), however hearts were significantly heavier in

27 Pik3r1 ${ }^{\text {WT/Y657X }}$ mice (Figure 1J) whether analysed with respect to lean mass or whole 
1 body weight by ANCOVA. No significant difference in either food consumption or

2 energy expenditure was seen at 16 weeks old on chow diet (Figure 1K,L). A similar

3 pattern of differences was seen between heterozygous and wild-type female mice for

4 all variables assessed in both sexes (Supplemental Figure S4).

\section{Reproductive function of Pik3r1 ${ }^{\text {WT/Y657X }}$ mice}

Postpubertal women with SHORT syndrome commonly exhibit anovulation

8 with severe ovarian hyperproduction of testosterone (25). This is most likely a

9 consequence of systemic severe insulin resistance, however important roles for

10 PI3K in the ovary are also known, with deletion of PTEN, a negative regulator of

$11 \mathrm{PI} 3 \mathrm{~K}$, in oocytes in mice producing unrestrained maturation of primordial follicles and

12 thus rapid exhaustion of the follicular pool (29). We thus assessed ovarian

13 morphology and reproductive function in the Pik3r1WT/Y657X mice. No significant

14 difference was seen in litter frequency for any pairwise genotype combination,

15 assessed between 10 and 18 weeks, however litter size was reduced significantly for

16 Pik3r1WTYY657X mothers whether mating with wild-type or heterozygous males, and

17 taking into account the reduced litter size expected in double heterozygous matings

18 due to homozygote non-viability (Supplemental Table S1). There was also a

19 surprising excess of heterozygous offspring both in double heterozygous crosses

20 and when make heterozygotes were crossed with wil-type females, but notwhen

21 wild-type males were crossed with heterozygous females. As expected, corpora

22 lutea were observed in both Pik3r1WT/Y657X and wild-type females of reproductive age

23 (at 42 days old), consistent with ovulation, and serum testosterone concentrations

24 were similar in both genotypes at 12 weeks old $(0.18 \pm 0.05 \mu \mathrm{g} / \mathrm{l}$ in heterozygotes

$25(\mathrm{n}=7)$ vs $0.23 \pm 0.04$ in wild-types $(\mathrm{n}=10)$ (Not significant)). More detailed

26 morphometric analysis of ovaries at 7 days old, before sexual maturation, showed a

27 grossly normal microscopic appearance, with the proportions and sizes of follicles at 
1 different developmental stages similar in wild type and heterozygous ovaries,

2 suggesting normal early follicle development (Figure 2).

4 Adipose response of Pik3r1 ${ }^{W T / Y 657 X}$ mice to a high fat diet

Lipodystrophy, or impaired accretion of adipose tissue, is a common feature of

6 SHORT syndrome (24), and the previously reported Pik3r1WT/R649W mouse was also

7 shown to have reduced subcutaneous adipose tissue even on chow diet. On the

8 other hand at least one human proband with the Y657X mutation showed normal

9 adipose development (25). To assess whether placing a greater load on adipose

10 tissue storage in Pik3r1WT/Y657X mice would unmask a lipodystrophic phenotype, mice

11 were fed a palatable $45 \%$ high fat diet (HFD) from 8 weeks old. Pik3r1WT/Y657X mice

12 showed significantly reduced gain of weight and whole body fat content under these

13 conditions over the 8 week period studied (Figure 3A,B). Epididymal white adipose

14 tissue was most severely affected (Figure $3 \mathbf{C}$ ), with the difference in inguinal

15 adipose tissue not achieving statistical significance by ANCOVA (Figure 3D). Brown

16 adipose tissue mass was unaffected (Figure 3E). Consistent with reduced adiposity,

17 Pik3r1WT/Y657X mice had lower serum leptin concentration in the fed state $(37.7 \pm 7.3 \mathrm{vs}$

$\left.1811.3 \pm 2.0 \mu \mathrm{g} / \mathrm{L}\left(\mathrm{n}=15,11 ; \mathrm{p}=3 \times 10^{-3}\right)\right)$, although this was not significant on fasting

19 (15.9 \pm 3.9 vs $10.3 \pm 2.2 \mu \mathrm{g} / \mathrm{L}(\mathrm{n}=13,13$; not significant)). Lean mass did not increase at

20 a greater rate in Pik3r1WT/Y657X mice than wild-type littermates (Supplemental Figure

21 S5), and liver weights also did not diverge, while heart weights were elevated in 22 heterozygotes, as on chow (Figure 3F,G).

23 Despite the marked decrease in weight of epididymal white adipose tissue, the 24 histological appearance and adipocyte size distribution was similar in both 25 genotypes (Figure $\mathbf{3} \mathbf{H}, \mathbf{I}$ ), indicating a smaller number of normal sized adipocytes in 26 heterozygotes. Ex vivo differentiation of preadipocytes from either inguinal or 27 epididymal depots using conventional protocols was normal, however (Figure $3 \mathbf{J}$ 
1 and Supplemental Figure S6) as previously reported for Pik3r1WT/R649W mice,

2 suggesting that the reduced adipose expansion is not accounted for by a cell

3 autonomous defect in differentiation capacity. To assess whether the impaired

4 adipose expansion might instead reflect altered energy homeostasis, food intake and

5 energy expenditure were next assessed. Pik3r1WT/Y657X mice, at odds with the

6 observed reduction in white adipose tissue expansion on high fat feeding, exhibited

7 significantly increased food intake (Figure 3K). Energy expenditure was also

8 increased, however (Figure 3L), possibly in part due to increased locomotor activity

9 during the dark cycle, although observed activity was not significantly increased

10 across the whole 24 hour cycle (Figure 3M).

\section{Pik3r1 ${ }^{W T / Y 657 X}$ mice are severely insulin resistant}

In accord with the critical role known to be played by PI3K in insulin action,

14 severe insulin resistance is common in SHORT syndrome, and insulin resistance

15 was also reported in Pik3r1WT/R649W mice. Assessed at 12 weeks old, neither male

16 nor female Pik3r1WT/Y657X mice on chow were hyperglycemic compared to wild-type

17 littermates, but plasma insulin concentrations were significantly raised, more severely so in the fed state, as in human severe insulin resistance (Table 1,

19 Supplemental Table S2). On high fat feeding male Pik3r1WT/Y657X mice remained

20 hyperinsulinemic, but the difference between mutant and control animals was

21 abolished as wild-type mice exhibited a much greater increase in insulin

22 concentrations on high fat feeding than Pik3r1WT/Y657X animals. Glucose

23 concentrations were slightly lower in the fed state on high fat diet in the

24 heterozygous mice than controls, which may also have contributed to the loss of a significant difference in insulin concentrations. Plasma adiponectin concentrations in

26 fasted chow-fed Pik3r1WT/Y657X mice were lower than in wild-type controls, however

27 no significant difference in concentrations were seen between genotypes in the other

28 conditions despite severe insulin resistance of heterozygous mice (Table 1). 
1 Moreover in Pik3r1WT/Y657X animals on HFD, adiponectin normalised to body fat

2 content was significantly higher than in controls (Supplemental Figure S7).

3 Normalised plasma leptin concentrations, in contrast, were lower in heterozygous

4 mice than controls (Supplemental Figure S7).

To evaluate insulin sensitivity in more detail, hyperinsulinemic euglycemic clamps were undertaken on both chow-fed or HFD-fed male mice. Initial clamps undertaken without isotopic tracers demonstrated that on chow feeding Pik3r1WT/Y657X

8 mice required a glucose infusion rate at steady state of only one eighth of the rate

9 required by wild-type mice, confirming severe insulin resistance (Figure 4A). On

10 HFD Pik3r1WT/Y657X mice remained extremely insulin resistant, however the difference

11 between heterozygous and wild-type mice was much smaller than on chow due to

12 insulin resistance induced in wild-type animals (Figure 4A). Further studies were

13 thus undertaken on chow. Glucose and insulin excursions on oral glucose tolerance

14 testing showed only a trend towards an increase in male Pik3r1WT/Y657X mice (Figure

15 4B,C), but were significantly increased in female mice (Supplemental Figure S8),

16 while the hypoglycemic response to insulin was clearly attenuated on insulin

17 tolerance testing in both sexes (Figure 4D and Supplemental Figure S8). Further

18 clamp studies in males incorporating isotopic tracers showed glucose disposal to be

$1919 \%$ lower in Pik3r1WT/Y657X mice (Figure 4E) while suppression of hepatic glucose

20 production by hyperinsulinemia was $49 \%$ lower compared to wild type littermates

21 (Figure 4F). Despite this, liver glycogen content was similar between heterozygous

22 Pik3r1WT/Y657X mice and wild type littermates in both fed and fasted states (Figure

23 4G). Insulin infusion lowered plasma free fatty acid concentration in plasma by $38 \%$

24 in wild-type mice, however this suppression was nearly abolished in heterozygous

25 mutant animals, consistent with impaired suppression of triglyceride lipolysis in 26 adipose tissue (Figure $\mathbf{4 H}$ ).

27 Intraperitoneal injection of insulin in male mice strongly induced Akt Ser 473 28 phosphorylation in liver, skeletal muscle and both epididymal and inguinal adipose 
1 tissue of wild-type controls, as expected, and this was reduced by $55 \%, 20 \%, 37 \%$

2 and $30 \%$ respectively in male Pik3r1WT/Y657X mice (Figure $4 \mathrm{I}-\mathrm{L}$ ). A similar pattern was

3 seen for threonine 308 phosphorylation (Supplemental Figure S9). As muscle

4 insulin sensitivity was previously reported not to be reduced in Pik3r1WT/R649W mice,

5 insulin responsiveness of soleus and extensor digitorum longus (EDL) muscles was

6 further assessed ex vivo. Soleus and EDL muscle from Pik3r1WT/Y657X mice both

7 showed a 1.3-fold reduction in insulin-stimulated deoxyglucose uptake compared to

8 wild-type muscle (Figure $\mathbf{4 M}$ ). Insulin-induced Akt phosphorylation was also

9 markedly reduced in both types of muscle ex vivo to a much more significant degree

10 than in vivo (Figure $\mathbf{4 N}, \mathbf{O})$.

11 Pik3r1 ${ }^{\text {WT/Y657X }}$ mice are hypolipidemic

12 Having shown that, like humans with SHORT syndrome, heterozygous

13 Pik3r1WT/Y657X mice show severe insulin resistance and lipodystrophy, we next

14 assessed whether, like humans, they are protected from fatty liver and metabolic

15 dyslipidaemia. Strikingly, in both fed and fasting states, and on either chow or high

16 fat diets Pik3r1WT/Y657X mice showed hypolipidemia, with lower plasma total

17 cholesterol and HDL cholesterol (Table 1). Plasma triglyceride concentration was

18 also lower in heterozygous animals in all cases except the fasting state after high fat

19 feeding, and plasma free fatty acid concentration in all cases except chow fed

20 animals in the fed state. A similar pattern of hypolipidemia was seen in chow fed

21 female mice in the fasting state (Table S2). Importantly no difference was seen in

22 immunoassay-determined VLDL concentrations in either chow fed or high fat diet fed

23 mice (Table 1).

$24 \quad$ No difference was seen in liver triglyceride content between heterozygous and 25 wild-type mice either on chow diet or on HFD, assessed both by Oil Red O staining 26 and biochemical quantification (Figure 5A,B), in keeping with the lack of difference 27 in liver weights previously noted. Liver cholesterol content was also the same in 28 chow-fed animals of both genotypes (Figure 5C). To screen for abnormal lipid 
1 absorption or handling by the intestine oral lipid tolerance testing was undertaken,

2 but although lower plasma triglyceride concentrations were seen at all time points,

3 the excursion after lipid loading was the same in both genotypes (Figure 5D), and

4 bomb calorimetry of faeces on chow showed the same energy content in both

5 groups (Figure 5E). Collectively these findings argue strongly against abnormal

6 intestinal lipid absorption and/or mobilisation as the explanation for hypolipidemia

7 and failure to gain adipose mass on HFD.

$8 \quad$ Srebp1 and Srebp2 are critical transcriptional regulators of de novo lipogenesis

9 and cholesterol synthesis respectively, and many studies have demonstrated that

10 they are activated by insulin in a PI3K-dependent manner. Their mRNA expression,

11 together with expression of a panel of Srebp1 target genes (Acc1, Fasn, Scd1) and

12 Srebp2 target genes (Mvk, Nsdhl), were assessed in the liver of chow fed animals in

13 the fed, fasting and refed states (Figure 5F-L). After 16 hours of fasting there was a

14 significant decrease in expression of all genes except Scd1 in Pik3r1WT/Y657X mice,

15 however no difference was seen either in the ad libitum fed state or after 6 hours of

16 refeeding. Moreover expression of all genes except Scd1 was much higher in both

17 fed and refed states than in fasting, raising questions about the relevance of the

18 prolonged fasting state to the observed hypolipidemic phenotype of ad lib fed

19 animals.

20 Other candidate transcriptional regulators relevant to hypolipidemia include 21 carbohydrate response element binding protein (Chrebp) and Xbp1, with the latter

22 having been shown to bind $p 85 \alpha$, facilitating its nuclear translocation $(19,20,26) \mathrm{A}$

23 sentinel Chrebp-responsive gene (Pklr) showed mild but significant repression in the

24 fasting state, but two Xbp1 target genes (Acacb and Dgat2) showed no

25 transcriptional repression during a fasting refeeding cycle (Supplementary Figure

26 S10). It has recently been proposed that acute insulin and Foxo-mediated

27 modulation of the ratio between Glucose-6-phosphatase (G6pc) and Glucokinase

28 (Gck) serves to toggle the direction of hepatocyte glucose flux between lipogenesis 
1 and hepatic glucose production, and that this mechanism is operative before

2 transcriptional changes in canonical lipogenic transcription factors are observed (30).

3 However although an increase in G6pc:Gck liver transcript levels was seen on

4 fasting of wild-type mice in this study, consistent with this model, this ratio was

5 suppressed in Pik3r1WT/Y657X mice, as reported in mice fed a Western-type diet (30).

6

\section{Discussion}

$8 \quad$ PIK3R1 was first sequenced in the 1990s and since then it has been

9 intensively studied in different cellular contexts, with many different constitutional

10 and conditional murine genetic models generated. These studies have collectively

11 established that PIK3R1 gene products are critically involved in signaling

12 downstream from tyrosine kinase receptors and also that they serve to integrate this

13 role with other signaling inputs, most commonly through interaction of relevant

14 proteins with the $\mathrm{N}$ terminal domain of $\mathrm{p} 85 \alpha$, which is truncated in the splice variants

$15 \mathrm{p} 55 \alpha$ and $\mathrm{p} 50 \alpha$. PIK3R1 gene products may also play signaling roles independent

16 of RTKs.

17 Human pathogenic mutations in PIK3R1 have only been discovered relatively

18 recently. Selective loss of p85 $\alpha$ produces agammaglobulinemic immunodeficiency

19 (10), closely replicating findings in the earlier, corresponding murine model (9), while

20 somatic activating mutations are found in some solid malignancies (31), in line with

21 the role of $\mathrm{PI} 3 \mathrm{~K}$ in transducing growth factor signaling. Germline activating

22 mutations, however, produce immunodeficiency due to impaired lymphocyte

23 maturation $(32,33)$, with no consistent metabolic or growth abnormalities described.

24 SHORT syndrome, the last disorder to be associated with PIK3R1 mutations, is

25 predominantly associated with mutations in the $\mathrm{C}$ terminal $\mathrm{SH} 2$ domain of

$26 \mathrm{p} 85 \alpha / \mathrm{p} 55 \alpha / \mathrm{p} 50 \alpha(21-23)$, and provides arguably the most surprising and yet

27 instructive insights into PIK3R1 function in humans, particularly in view of discordant 
1 phenotypic features between it and the various murine Pik3r1 knockout models that

2 have been reported.

Another mouse model of SHORT syndrome has recently been described,

4 heterozygous for the common SHORT-associated R649W mutation in the

5 phosphotyrosine binding motif of the $\mathrm{C}$ terminal $\mathrm{SH} 2$ domain (26). This model

6 recapitulated key features of SHORT syndrome including growth impairment,

7 lipodystrophy and insulin resistance. While the phenotype of the novel model we

8 describe is broadly concordant this model, it differs in some significant respects, and

9 we moreover extend phenotyping with more detailed developmental assessment and

10 challenge with a high fat diet.

11 As previously described for the R649W mutation, no mice homozygous for the

12 Pik3r1 Y657X mutation were born, contrasting with Pik3r1 knockout mice which are

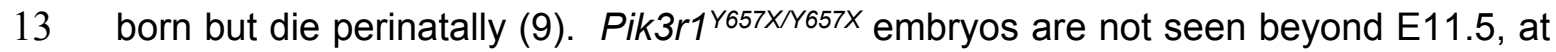

14 which stage they are smaller and retarded in development compared to wild-type

15 and heterozygous embryos. This timing is reminiscent of mice with knockout of both

16 Pik3r1 and Pik3r2, which do not survive beyond E12.5 (15). Homozygous knockout

17 or homozygosity for a kinase dead mutant of $p 110 \alpha$ also both lead to lethality around

$18 \mathrm{E} 10.5(5,34)$, while embryos without functional $\mathrm{p} 110 \delta$ are viable $(35)$, and those

19 without functional $\mathrm{p} 110 \beta$ have been reported to have variable phenotypes (8).

20 These findings demonstrate that the SHORT-related allele is not simply a loss-of-

21 function allele, but that instead it disrupts Pik3r1 function in a manner that renders it

22 resistant to rescue by other PI3K regulatory subunits. Pertinent to this are prior

23 findings that expression of $p 85 \beta$ is upregulated and expression of all three $p 110$

24 catalytic subunits downregulated in cells from p85 $\alpha$ knockout mice $(9,36,37)$. In

25 Pik3r1WT/Y657X mice, as in dermal fibroblasts from humans with SHORT syndrome

26 (25), p85 $\beta$ expression is unaffected, however $\mathrm{p} 110 \alpha$ was modestly downregulated in

27 subcutaneous adipose tissue only, and $\mathrm{p} 110 \beta$ more markedly downregulated in both 
1 white adipose tissue and liver. These findings suggest that it may be the failure to upregulate $p 85 \beta$ that is most important in the phenotypic differences between $p 85 \alpha$ knockout and Pik3r1WT/Y657X mice.

Reduced embryo size may be due to an embryo autonomous growth defect, to

5 impaired placental function, and/or to perturbed placental nutrient flux. Indeed,

6 recent studies of mice heterozygous for a kinase dead $\mathrm{p} 110 \alpha$ allele (Pik3cakD/WT $)$

7 have shown that $\mathrm{p} 110 \alpha$ action on both maternal and fetal sides of the placental

8 circulation influence each of these (28). Pik3r1WT/Y657X fetuses are around $20 \%$

9 smaller that wild-type fetuses at E15.5, which is similar to the decrease noted in

$10 P i k 3 c a^{K D / W T}$ fetuses previously. Although no significant reduction in placental mass

11 was seen, striking reduction of the vascularisation of the placental exchange region

12 was seen at E15.5, again very similar to the Pik3ca ${ }^{K D / W T}$ model. In contrast to that

13 model, however, the size of the placental exchange region and thickness of the

14 diffusion barrier were normal for Pik3r1WT/Y657X fetuses. These findings are consistent

15 with $\mathrm{p} 85 \alpha$ and $\mathrm{p} 110 \alpha$ co-operating in formation of the placental vascular tree, and

16 add to prior evidence highlighting the importance of class IA PI3Ks (particularly

$17 \mathrm{p} 110 \alpha)$ in developmental angiogenesis (38).

18 In the previous study of Pik3ca ${ }^{K D / N T}$ mice, maternal genotype as well as

19 fetoplacental genotype was important in determining placental phenotype. This was

20 not assessed directly in this study, where wild-type dams were used for

21 developmental studies, however a marked effect of maternal genotype was seen on

22 litter size, which were reduced by around $50 \%$ of the expected for heterozygous

23 dams, and apart from homozygous lethality no evidence of selection for fetal

24 genotype was apparent. Whether this relates to reduced size of the heterozygous

25 dams, to their metabolic state during gestation, or both, remains to be determined.

26 As previous studies have suggested that the insulin resistance of pregnancy is

27 caused by increased Pik3r1 expression (39), it will be interesting to assess whether 
1 pregnancy exaggerates the already severe insulin resistance seen in Pik3r1WT/Y657X

2 mice in the non pregnant state, and whether any such exacerbation influences

3 growth of the fetoplacental unit.

PI3K also plays several important roles in ovarian function, some of which,

5 such as the activation of dormant primordial follicles, are follicle autonomous and

6 some of which are indirect, mediated by factors such as systemic insulin resistance.

7 This is manifest in the severe hyperandrogenism and ovulatory dysfunction seen in

8 young women with SHORT syndrome (25). Despite these well established links, we

9 report no evidence of altered early follicle development, nor evidence of impaired

10 fertility nor hyperandrogenism in female Pik3r1WT/Y657X mice. This suggests either

11 that Pik3r1 gene products are not involved in triggering oocyte maturation, or that the

12 degree of PI3K hypofunction is insufficient to compromise this action. The lack of

13 features of insulin resistance-related ovulatory dysfunction and hyperandrogenism is

14 in keeping with many prior studies showing that mice model this highly prevalent

15 aspect of human severe insulin resistance poorly (40).

16 SHORT syndrome commonly features partial lipodystrophy $(24,25)$, and on

17 chow diet the previously reported Pik3r1WT/R649W mouse, too, was reported to have

18 reduced subcutaneous adipose depots (26). In this study no significant differences

19 in whole body fat content and adipose depot weights were observed on chow when

20 the smaller size of the Pik3r1WT/R649W mice was taken into account, however they

21 diverged strikingly between wild type and heterozygotes on high fat feeding. Ex vivo

22 differentiation of white preadipocytes was not impaired, consistent with the previous

23 study, although in preadipocyte cell lines overexpression of the mutant has been

24 shown to impair adipogenesis markedly (25). It was previously suggested that a

25 reduced number of preadipocytes in adipose tissue might account for reduced

26 adipose expansion, however although this was not assessed in the current study,

27 the normal adipocyte size and histological appearance argue against this, as a larger

28 adipocyte size would be expected if a smaller number of precursor cells were 
1 accommodating the same hypercaloric load. Alternative explanations for reduced

2 adipose accretion that are disproven by the current study are reduced food intake or

3 intestinal lipid malabsorption, or increased partitioning of excess energy into growth

4 of non adipose tissues.

5 Lipodystrophy in humans and mice is normally associated strongly with

6 exaggerated features of obesity-associated metabolic syndrome including fatty liver,

7 metabolic dyslipidaemia, and low plasma adiponectin concentrations (41, 42),

8 however SHORT syndrome is unique among human insulin resistant partial

9 lipodystrophies in featuring reduced adiposity with preserved plasma adiponectin

10 levels, absence of fatty liver and apparent protection from metabolic dyslipidemia

$11(24,25)$. This is similar to the profile seen in people with severe insulin resistance

12 due to mutations in the insulin receptor gene (43), suggesting that proximal insulin

13 signalling defects in humans produce a distinct subphenotype of insulin resistance.

14 The mechanisms underlying these human observations have not been tested,

15 however insulin resistance has been uncoupled from hyperlipidemia in various

16 mouse models, including mice with liver-specific knockout of Insr (44), both Irs1 and

17 Irs2 (45), Pik3ca (46) or Akt2 (47). These severe perturbations have generally been

18 suggested to produce hypolipidemia through loss of insulin-stimulated upregulation

19 of genes involved in de novo lipogenesis and/or cholesterol synthesis. The recent

20 Pik3r1WT/R649W SHORT syndrome model mice, in contrast, were said to have normal

21 blood lipid concentrations, however in the case of the Pik3r1WTTY657X mouse we report

22 hypolipidemia in both males and females, on chow diet and high fat feeding, and in

23 both the fed and fasted state. Moreover liver triglyceride levels showed no

24 difference between wild-type and Pik3r1WT/Y657X mice, although in the face of

25 lipodystrophic severe insulin resistance they would normally be increased. This

26 suggests that this murine model may permit dissection of mechanisms underlying

27 the dyslipidemia-insulin resistance dissociation. 
2 mutation in a feed/fasting/refeeding paradigm did show significant reduction in

3 transcript levels of Srebp and their transcriptional targets in the liver, with a sentinel

4 Chrebp-responsive gene also mildly suppressed, however these changes were only

5 seen in the fasting state. Whether these mild fasting changes can explain the

6 hypolipidemia observed, which is most striking in the fed state on high fat diet, is

7 unclear. No evidence was found for reduced lipogenic transcriptional activity of

$8 X b p 1$, which has both been shown to interact with $p 85 \alpha(18,19)$, and to serve as a

9 potent lipogenic transcription factor (20). A further mechanism toggling glucose

10 routing between hepatic lipogenesis and glucose secretion depends on the relative

11 expression of glucose-6-phosphatase (G6pc) and glucokinase (Gck), with a high

12 G6pc:Gck ratio favouring hepatic glucose output and disfavouring lipogenesis (30).

13 However although the refed G6pc:Gck ratio did significantly differ in Pik3r1WT/Y657X

14 mice compared to controls, the direction of the difference would be predicted to

15 favour lipogenesis.

16 Interestingly, global knockout of the insulin-responsive Glut4 transporter in

17 mice grossly mimics the phenotype of the mice we describe in key respects,

18 featuring insulin resistance, severely reduced adipose tissue, and an enlarged heart

19 (48). Moreover, although fatty liver was not observed, blood lipid levels and liver

20 triglyceride secretion was markedly increased (49), but was countered by

21 accelerated VLDL clearance by peripheral tissues, demonstrating extensive

22 remodelling of metabolism (50). In this case lipid tolerance testing does not provide

23 evidence for similarly enhanced clearance of lipid, however. Gaining further insight

24 into the mechanisms uncoupling dyslipidemia from insulin resistance in Pik3r1WT/Y657X

25 mice will require metabolic flux studies, and consideration of the possibility that

26 effects on hepatic metabolism are effected by primary dysfunction in other insulin

27 sensitive organs such as adipose tissue, which has recently been shown in some

28 contexts to be critical for such indirect regulation ofhepatic gluconeogenesis $(51,52)$. 
In summary, we describe a novel mouse model of SHORT syndrome driven by

2 a naturally occurring human mutation in PIK3R1. We describe novel developmental,

3 reproductive and metabolic aspects of the model, and establish that it recapitulates

4 the highly unusual human observation of lipodystrophic insulin resistance uncoupled

5 from atherogenic dyslipidaemia.

6

\section{METHODS}

\section{$8 \quad$ Mice generation and maintenance}

A vector containing the Pik3r1 exon 15 Y $657 \mathrm{X}$ mutation and a neomycin

10 resistance cassette flanked by LoxP sites was introduced into the genome of

11 C57BI/6 embryonic stem cells by electroporation and homologous recombination.

12 Targeted cells were injected into BI/6J blastocysts to generate chimeras which were

13 bred onto the $\mathrm{C} 57 \mathrm{BI} / 6 \mathrm{~J}$ background to create the mutant strain (Supplemental

14 Figure S1). All animals were kept on a C57BI/6J background, backcrossed at least

15 three times, and housed on a 12-hour-light/12-hour-dark cycle at $23^{\circ} \mathrm{C}$, with ad

16 libitum access to water and food. Feed was with either chow (Catalogue no. 105,

17 Safe diets) or a $45 \%$ high fat diet (45\% fat, $35 \%$ Carbohydrate and $25 \%$ protein)

18 (Catalogue no. D12451, Research diet, Inc). All animal experiments were carried out

19 under the UK Home Office Animals (Scientific Procedures) Act 1986, following

20 ethical review by the University of Cambridge.

\section{Fetoplacental development studies}

Single male mice were housed with a female mouse and noon of the day of detection of a vaginal plug was taken to be E0.5. Images of dissected embryos were acquired with a Zeiss SteREO Discovery V8 microscope and AxioCam MRc5 camera. Placentas were bisected and one half was fixed in $4 \%$ (wt/vol)

26 paraformaldehyde, paraffin-embedded, exhaustively sectioned at $7 \mu \mathrm{m}$ and stained

27 with hematoxylin and eosin to analyse gross placental structure. The other half was

28 fixed in 4\% (wt/vol) glutaraldehyde, embedded in Spurr's epoxy resin and a single, 
1 and $1 \mu \mathrm{m}$ midline section was cut and then stained with toluidine blue for detailed

2 analysis of labyrinthine (exchange) zone structure using the Computer Assisted

3 Stereological Toolbox (CAST v2.0) program as previously described (28).

4 Assessment of growth, body composition, and energy homeostasis

Fat and lean mass of live mice was determined by time-domain NMR using a

6 Bruker's minispec whole body composition analyser. For determination of food

7 intake and energy expenditure, male mice were acclimatized for 1 week to single

8 housing then given $200 \mathrm{~g}$ of standard chow diet or $45 \%$ high fat diet for 10 days.

9 Food intake was measured every 24 hours, and energy expenditure was measured

10 by indirect calorimetry as described previously (53). Locomoter activity was

11 quantified as beam breaks over 48 hours. Fecal energy content was measured by

12 drying then totally combusting feces from $48 \mathrm{hr}$ calorimeter runs in an IKA

13 Calorimeters Oxygen Bomb calorimeter (IKA C1).

\section{Biochemical assays}

15 Blood was collected by cardiac puncture and plasma separated and snap

16 frozen immediately. Plasma analytes were assayed following manufacturers'

17 instructions with the following assays: glucose (Siemens Healthcare Diagnostics),

18 insulin (Meso Scale Discovery), leptin (Meso Scale Discovery), adiponectin (Meso

19 Scale Discovery), triacylglyceride (Siemens Healthcare Diagnostics), total

20 cholesterol (Siemens Healthcare Diagnostics), HDL cholesterol (Randox), VLDL

21 (Cusabio), free fatty acids (Roche) and testosterone (IBL international). Plasma

22 insulin concentration during glucose tolerance testing used an ELISA (Crystal

23 Chem).

24 Hepatic glycogen content was determined in snap-frozen liver samples that 25 were weighed before glycogen hydrolysis in $1 \mathrm{M} \mathrm{HCl}\left(2.5 \mathrm{~h}\right.$ at $\left.100^{\circ} \mathrm{C}\right)$. Hydrolysate 26 was neutralized with $\mathrm{NaOH}$ and glucose units analysed fluorometrically as described 27 in (54). Hepatic triglyceride was extracted and measured with a Triglyceride 28 Colorimetric Assay kit (Cayman) according to the manufacturer's instructions. 
1 Hepatic cholesterol was extracted from $10 \mathrm{mg}$ liver using $400 \mathrm{uL}$ of

2 chloroform:isopropanol:NP-40 mixture (7:11:0.1) in a homogenizer before

3 centrifugation and air drying of superatant at $50^{\circ} \mathrm{C}$. After 30 minutes of vacuum

4 drying lipids was dissolved in PBS and cholesterol was assayed with Siemens

5 Dimension Healthcare following the manufacturer's instructions (Siemens

6 Healthcare Diagnostics).

$7 \quad$ In vivo metabolic studies

8 For fasting/refeeding studies, mice were fed ad libitum on the standard chow

9 until 16 weeks before fasting for 16 hours and refeeding with chow for 6 hours.

10 Tissues were harvested and snap-frozen before overnight fasting, after fasting and

11 then after 6 hour refeeding. For study of acute insulin action 16 hours fasted mice

12 (16 weeks old) were injected with $2 \mathrm{U} / \mathrm{kg}$ insulin intraperitoneally and tissues were

13 collected after 10 minutes and snap frozen in liquid nitrogen. Glucose tolerance

14 testing was undertaken on overnight (16 hour) fasted mice using $2 \mathrm{~g} / \mathrm{kg} 20 \%$ glucose

15 via oral gavage.For insulin tolerance testing mice were fasted for 6 hours and

16 challenged with $0.5 \mathrm{U} / \mathrm{kg}$ intraperitoneal insulin. For lipid tolerance testing overnight

1716 hour fasted mice were administered $10 \mathrm{~mL} / \mathrm{kg}$ olive oil by oral gavage.

18 Hyperinsulinemic euglycemic clamp studies and the subsequent sample

19 processing and analysis were conducted as described previously (55) with minor

20 modifications. 16 week old mice were fasted overnight, before clamps were carried

21 out with a priming dose of human insulin $(0.7 \mathrm{mU})$, followed by a constant insulin and

$22\left[3^{-3} \mathrm{H}\right]-\mathrm{D}-$ glucose tracer infusion at $7 \mathrm{mU} / \mathrm{h}$ and $0.72 \mu \mathrm{Ci} / \mathrm{h}$, respectively.

\section{Study of insulin action ex vivo}

24 Measurement of insulin action on isolated Soleus and Extensor Digitorum 25 Longus was undertaken in 16 week old male mice as previously described(56). For 26 primary preadipocyte studies fat pads from 8 week-old male mice were isolated, 27 minced and digested in pre-warmed sterile digest solution (1:3 BSA $7.5 \%$ 28 solution:Hanks' Buffered Salt Solution, and $1 \mathrm{mg} / \mathrm{mL}$ Collagenase Type II). Digested 
1 tissues were strained through $100 \mu \mathrm{m}$ cell strainers and placed on ice for 20

2 minutes. Following removal of the fat layer, supernatant was mixed 1:1 with growth

3 medium (high-glucose DMEM, 10\% NCS, 4 mM L-glutamine and $100 \mathrm{U} / \mathrm{L}$ penicillin

4 streptomycin), and the stromovascular fractions washed twice with growth medium,

5 and resuspended in differentiation medium (growth media supplemented with $2.4 \mathrm{nM}$

6 insulin and $150 \mu \mathrm{M}$ sodium L-ascorbate). The cells were plated into two wells of a

7 12-well plate and fed with fresh differentiation medium daily for 3 days and then

8 every other day until Oil-Red O staining as described previously(25).

\section{Histology}

10 Ovaries from day 7,21 or 42 (D7, D21 or D42) wildtype and heterozygous

11 female mice (3 per age per genotype) were fixed in $10 \%$ neutral buffered formalin 12 solution, dehydrated in ethanol, infiltrated with Histo-Clear, and embedded in

13 paraffin. $5 \mu \mathrm{m}$ serial sections were stained with haematoxylin and eosin. Follicles in 14 every $5^{\text {th }}$ section of D7 ovaries were classified morphologically as primordial, 15 transitional, primary, primary plus or secondary, and their cross-sectional area 16 measured in Image J(57). To assess ovulation, every $10^{\text {th }}$ section of the D42 ovaries 17 was analysed for the presence of corpora lutea.

Adipocyte size measurement was undertaken in formalin-fixed, paraffinembedded adipose tissue stained with haematoxylin and eosin. Cell sizes were 20 measured using CellP software (Olympus Scientific Solutions). At least 1000 cells 21 per genotype were analyzed. For oil-red-O staining of liver, livers were snap-frozen and embedded in optimum cutting temperature (OCT) media. Following sectioning 23 with a cryostat, sections were fixed in $10 \% \mathrm{NBF}$, incubated in $0.5 \%$ oil red $\mathrm{O}$ working solution, and counterstained with haematoxylin. Images were taken with an Olympus CKX41 inverted microscope and Olympus DP20 microscope camera.

\section{Gene expression analysis}

For protein expression studies, freshly dissected issues were snap-frozen in

28 liquid nitrogen. Liver and skeletal muscle were homogenised using MD ceramic 
1 beads and an MD machine in RIPA buffer with proteinase and phosphatase

2 inhibitors (Roche). Adipose tissue was ground using mortar and pestle in liquid

3 nitrogen before dissolving in the buffer. Protein concentrations were determined

4 using the BCA assay (BioRad). Western blotting employed the Novex gel system

5 (Thermo Fisher Scientific). Antibodies used are shown in Supplemental Table S3

6 Imaging and quantification of immunoblots were undertaken using the BioRad image

7 system.

8 For RNA expression studies total RNA was extracted by RNeasy kits (Qiagen).

$9500 \mathrm{ng}$ of total RNA was used for cDNA synthesis with the ImProm-Il reverse

10 transcription system (Promega). Realtime quantitative PCR was carried out in $13 \mu \mathrm{l}$

11 reaction volumes containing $300 \mathrm{nM}$ forward and reverse primers, $150 \mathrm{nM}$

12 fluorogenic probe when applicable, and ABI Taqman or SYBR® Green Master Mix

13 (Thermo Fisher Scientific). Reactions were conducted in duplicate with a standard

14 curve for each gene using the QuantStudio ${ }^{\mathrm{TM}} 7$ Flex Real-Time PCR system

15 (Thermo Fisher Scientific). Expression of target genes were normalised to the

16 geometric mean of 4 housekeeping genes (Ywhaz, Ppia, B2m and Eef1a1) (see

17 Supplemental Table S4 for primer and probe sequences).

18 Statistical Analysis

19 Numerical data are presented as mean \pm SEM and statistical tests used are

20 indicated in figure legends. In general unpaired two-tailed Student's $t$ tests were

21 used to compare two groups of data, while ANOVA with post hoc testing as indicated

22 was performed for more than two groups. All these analyses were performed using

23 GraphPad Prism (GraphPad Software). Tissue weights, food consumption and

24 energy expenditure were analyzed by ANCOVA using XLSTAT (Addinsoft), with total

25 body weight or lean mass as covariant.

27 Author Contributions 
1 Conceptualization, RKS, AK; Methodology, SV, JJ, PV; Formal Analysis, AK, IZ, SV,

2 IHD, PRT, DAB, DH, RK, SF, PV, AS-P, JJ, RKS; Investigation, AK, IZ, SV, IHD,

3 PRT, DAB, DH, RK, PV, AS-P, JJ, RKS; Writing - Original Draft, RKS, AK, IHD;

4 Writing - Review \& Editing, IZ, SV, PRT, DAB, DH, RK, AVP, SF, PV, AS-P, JJ, SO;

5 Supervision, RKS; Project Administration, RKS; Funding Acquisition, RKS, SO, AVP

6

7 Acknowledgements

8 RKS and SO were supported by the Wellcome Trust [grants WT098498 and

9 WT095515 respectively], and by the Medical Research Council (MRC) [grant

10 MC_UU_12012/5]. AVP and SV were funded by the British Heart Foundation [grant

$11 \mathrm{RG} / 12 / 13 / 29853]$ and the MRC [MC_UU_12012/2]. Animal work was conducted in

12 the MRC Disease Model Core [MC_UU_12012/5]. We are grateful for technical

13 assistance from Dr. Amy Warner at the MRC Disease Model Core, Mr Keith Burling

14 at the MRC MDU Mouse Biochemistry Laboratory, Mr Warner and Ms Phillips at the

15 Histology Core and Drs Brian Lam and Marcella Ma at the Genomics and

16 Transcriptomics core. All of them are funded by MRC under MRC_MC_UU_12012/5.

17 We would also like to thank Mr Gregory Strachan at the Imaging Core, which is

18 funded by the Wellcome Trust [Grant 100574/Z/12/Z] for assistance.

\section{References}

20 1. Vanhaesebroeck B, Stephens L, Hawkins P. PI3K signalling: the path to discovery

21 and understanding. Nat. Rev. Mol. Cell Biol. 2012;13(3):195-203.

22 2. Cantley LC. The Phosphoinositide 3-Kinase Pathway. Science.

23 2002;296(5573):1655-1657.

24 3. Samuels Y. High Frequency of Mutations of the PIK3CA Gene in Human Cancers.

25 Science. 2004;304(5670):554-554.

26 4. Mirzaa $\mathrm{G}$ et al. PIK3CA-associated developmental disorders exhibit distinct

27 classes of mutations with variable expression and tissue distribution. JCl Insight

28 2016;1(9):12-15.

29 5. Foukas LC et al. Critical role for the p110alpha phosphoinositide-3-OH kinase in

30 growth and metabolic regulation. Nature 2006;441(7091):366-70.

31 6. Knight ZA et al. A Pharmacological Map of the PI3-K Family Defines a Role for

32 p110a in Insulin Signaling. Cell 2006;125(4):733-747.

33 7. Angulo I et al. Phosphoinositide 3-Kinase Gene Mutation Predisposes to

34 Respiratory Infection and Airway Damage. Science. 2013;342(6160):866-871.

35 8. Guillermet-Guibert J et al. Novel Role for $\mathrm{p} 110 \beta$ PI 3-Kinase in Male Fertility

36 through Regulation of Androgen Receptor Activity in Sertoli Cells. PLoS Genet. 
2015;11(7):e1005304.

9. Fruman DA et al. Impaired B cell development and proliferation in absence of phosphoinositide 3-kinase p85alpha. Science 1999;283(5400):393-7.

10. Conley ME et al. Agammaglobulinemia and absent $B$ lineage cells in a patient lacking the p85a subunit of PI3K. J. Exp. Med. 2012;209(3):463-470.

11. Terauchi $Y$ et al. Increased insulin sensitivity and hypoglycaemia in mice lacking the p85 alpha subunit of phosphoinositide 3-kinase. Nat. Genet. 1999;21(2):230-5. 12. Mauvais-Jarvis F et al. Reduced expression of the murine p85alpha subunit of phosphoinositide 3-kinase improves insulin signaling and ameliorates diabetes. J. Clin. Invest. 2002;109(1):141-9.

13. Chen D et al. p50 /p55 Phosphoinositide 3-Kinase Knockout Mice Exhibit Enhanced Insulin Sensitivity. Mol. Cell. Biol. 2004;24(1):320-329.

14. Fruman DA et al. Hypoglycaemia, liver necrosis and perinatal death in mice lacking all isoforms of phosphoinositide 3-kinase p85 alpha. Nat. Genet. 2000;26(3):379-382.

15. Brachmann SM et al. Role of phosphoinositide 3-kinase regulatory isoforms in development and actin rearrangement. Mol. Cell. Biol. 2005;25(7):2593-606.

16. Ueki K et al. Molecular Balance between the Regulatory and Catalytic Subunits of Phosphoinositide 3-Kinase Regulates Cell Signaling and Survival. Mol. Cell. Biol. 2002;22(3):965-977.

17. Geering B, Cutillas PR, Nock G, Gharbi SI, Vanhaesebroeck B. Class IA phosphoinositide 3-kinases are obligate p85-p110 heterodimers. Proc. Natl. Acad. Sci. U. S. A. 2007;104(19):7809-14.

18. Winnay JN, Boucher J, Mori MA, Ueki K, Kahn CR. A regulatory subunit of phosphoinositide 3-kinase increases the nuclear accumulation of X-box-binding protein-1 to modulate the unfolded protein response. Nat. Med. 2010;16(4):438-445. 19. Park SW et al. The regulatory subunits of PI3K, p85 $\alpha$ and $p 85 \beta$, interact with XBP-1 and increase its nuclear translocation. Nat. Med. 2010;16(4):429-437.

20. Lee A-H, Scapa EF, Cohen DE, Glimcher LH. Regulation of Hepatic Lipogenesis by the Transcription Factor XBP1. Science. 2008;320(5882):1492-1496.

21. Chudasama KK et al. SHORT syndrome with partial lipodystrophy due to impaired phosphatidylinositol 3 kinase signaling. Am. J. Hum. Genet. 2013;93(1):150-157.

22. Dyment DA et al. Mutations in PIK3R1 cause SHORT syndrome. Am. J. Hum. Genet. 2013;93(1):158-166.

23. Thauvin-Robinet $C$ et al. PIK3R1 mutations cause syndromic insulin resistance with lipoatrophy. Am. J. Hum. Genet. 2013;93(1):141-149.

24. Avila M et al. Clinical reappraisal of SHORT syndrome with PIK3R1 mutations: Toward recommendation for molecular testing and management. Clin. Genet. 2016;89(4):501-506.

25. Huang-Doran I et al. Insulin resistance uncoupled from dyslipidemia due to Cterminal PIK3R1 mutations. JCl insight 2016;1(17):e88766.

26. Winnay JN et al. PI3-kinase mutation linked to insulin and growth factor resistance in vivo. J. Clin. Invest. 2016;126(4):1401-1412.

27. Ko HR et al. P42 Ebp1 regulates the proteasomal degradation of the p85 regulatory subunit of $\mathrm{PI} 3 \mathrm{~K}$ by recruiting a chaperone-E3 ligase complex HSP70/CHIP. Cell Death Dis. 2014;5(3):e1131.

28. Sferruzzi-Perri AN, López-Tello J, Fowden AL, Constancia M. Maternal and fetal genomes interplay through phosphoinositol 3-kinase(PI3K)-p110 $\alpha$ signaling to modify placental resource allocation. Proc. Natl. Acad. Sci. 2016;113(40):1125511260.

29. Reddy $P$ et al. Oocyte-Specific Deletion of Pten Causes Premature Activation of the Primordial Follicle Pool. Science. 2008;319(5863):611-613.

30. Haeusler RA et al. Integrated control of hepatic lipogenesis versus glucose production requires FoxO transcription factors. Nat. Commun. 2014;5:5190. 
31. Jaiswal BS et al. Somatic Mutations in p85a Promote Tumorigenesis through Class IA PI3K Activation. Cancer Cell 2009;16(6):463-474.

32. Lucas CL et al. Heterozygous splice mutation in PIK3R1 causes human immunodeficiency with lymphoproliferation due to dominant activation of PI3K. J. Exp. Med. 2014;211(13):2537-2547.

33. Deau MC et al. A human immunodeficiency caused by mutations in the PIK3R1 gene. J. Clin. Invest. 2014;124(9):3923-3928.

34. Bi L, Okabe I, Bernard DJ, Wynshaw-Boris A, Nussbaum RL. Proliferative defect and embryonic lethality in mice homozygous for a deletion in the $\mathrm{p} 110 \alpha$ subunit of phosphoinositide 3-kinase. J. Biol. Chem. 1999;274(16):10963-10968.

35. Okkenhaug $\mathrm{K}$ et al. Impaired B and T Cell Antigen Receptor Signaling in p110delta PI 3-Kinase Mutant Mice. Science. 2002;297(August):1031-1034. 36. Suzuki $\mathrm{H}$ et al. Xid-like immunodeficiency in mice with disruption of the p85alpha subunit of phosphoinositide 3-kinase. Science 1999;283(5400):390-2.

37. Lu-Kuo JM, Fruman DA, Joyal DM, Cantley LC, Katz HR. Impaired Kit- but Not Fcepsilon RI-initiated Mast Cell Activation in the Absence of Phosphoinositide 3Kinase p85alpha Gene Products. J. Biol. Chem. 2000;275(8):6022-6029. 38. Graupera $\mathrm{M}$ et al. Angiogenesis selectively requires the $\mathrm{p} 110 \mathrm{alpha}$ isoform of PI3K to control endothelial cell migration. Nature 2008;453(7195):662-666. 39. Barbour $L$ a et al. Human placental growth hormone increases expression of the p85 regulatory unit of phosphatidylinositol 3-kinase and triggers severe insulin resistance in skeletal muscle. Endocrinology 2004;145(3):1144-1150. 40. Huang-Doran I, Franks S. Genetic Rodent Models of Obesity-Associated Ovarian Dysfunction and Subfertility: Insights into Polycystic Ovary Syndrome. Front. Endocrinol. (Lausanne). 2016;7(June):53.

41. Savage DB. Mouse models of inherited lipodystrophy. Dis Model Mech 2009;2(11-12):554-562.

42. Semple RK, Savage DB, Cochran EK, Gorden P, O'Rahilly S. Genetic syndromes of severe insulin resistance. Endocr. Rev. 2011;32(4):498-514. 43. Semple RK et al. Postreceptor insulin resistance contributes to human dyslipidemia and hepatic steatosis. J. Clin. Invest. 2009;119(2):315-322. 44. Biddinger SB et al. Hepatic Insulin Resistance Is Sufficient to Produce Dyslipidemia and Susceptibility to Atherosclerosis. Cell Metab. 2008;7(2):125-134. 45. Guo $S$ et al. The Irs 1 branch of the insulin signaling cascade plays a dominant role in hepatic nutrient homeostasis. Mol. Cell. Biol. 2009;29(18):5070-83.

46. Sopasakis VR et al. Specific Roles of the p110a Isoform of Phosphatidylinsositol 3-Kinase in Hepatic Insulin Signaling and Metabolic Regulation. Cell Metab. 2010;11(3):220-230.

47. Leavens KF, Easton RM, Shulman GI, Previs SF, Birnbaum MJ. Akt2 Is Required for Hepatic Lipid Accumulation in Models of Insulin Resistance. Cell Metab. 2009;10(5):405-418.

48. Katz EB, Stenbit AE, Hatton K, DePinho R, Charron MJ. Cardiac and adipose tissue abnormalities but not diabetes in mice deficient in GLUT4. Nature 1995;377(6545):151-155.

49. Ranalletta M et al. Altered Hepatic and Muscle Substrate Utilization Provoked by GLUT4 Ablation. Diabetes 2005;54(April):935-943.

50. Kotani K, Peroni OD, Minokoshi Y, Boss O, Kahn BB. GLUT4 glucose transporter deficiency increases hepatic lipid production and peripheral lipid utilization. J. Clin. Invest. 2004;114(11):1666-1675.

51. Perry RJ et al. Hepatic acetyl CoA links adipose tissue inflammation to hepatic insulin resistance and type 2 diabetes. Cell 2015;160(4):745-758.

52. Titchenell PM et al. Direct Hepatocyte Insulin Signaling Is Required for Lipogenesis but Is Dispensable for the Suppression of Glucose Production. Cell Metab. 2016;23(6):1154-1166.

53. Whittle AJ et al. Soluble LR11/SorLA represses thermogenesis in adipose tissue 
1 and correlates with BMI in humans. Nat. Commun. 2015;6:8951.

2 54. Lowry OH, Passonneaau J V. A flexible system of enzyme analysis. Academic

3 Press; 1972:

$4 \quad 55$. Schreiber R et al. Hypophagia and metabolic adaptations in mice with defective

5 ATGL-mediated lipolysis cause resistance to HFD-induced obesity. Proc. Natl. Acad.

6 Sci. U. S. A. 2015;112(45):13850-5.

7 56. Sell H, Jensen J, Eckel J. Measurement of Insulin Sensitivity in Skeletal Muscle

8 In Vitro. Methods Mol Biol 2012;933:255-263.

9 57. Da Silva-Buttkus $P$ et al. Effect of cell shape and packing density on granulosa

10 cell proliferation and formation of multiple layers during early follicle development in

11 the ovary. J. Cell Sci. 2008;121(23):3890-3900.

12 
bioRxiv preprint doi: https://doi.org/10.1101/224485; this version posted November 24,2017 . The copyriaht holder for this preprint (which was not certified by peer review) is the author/funder, who has granted bioRxiv a license to display the preprint in perpetuity. It is made available under aCC-BY 4.0 International license. 


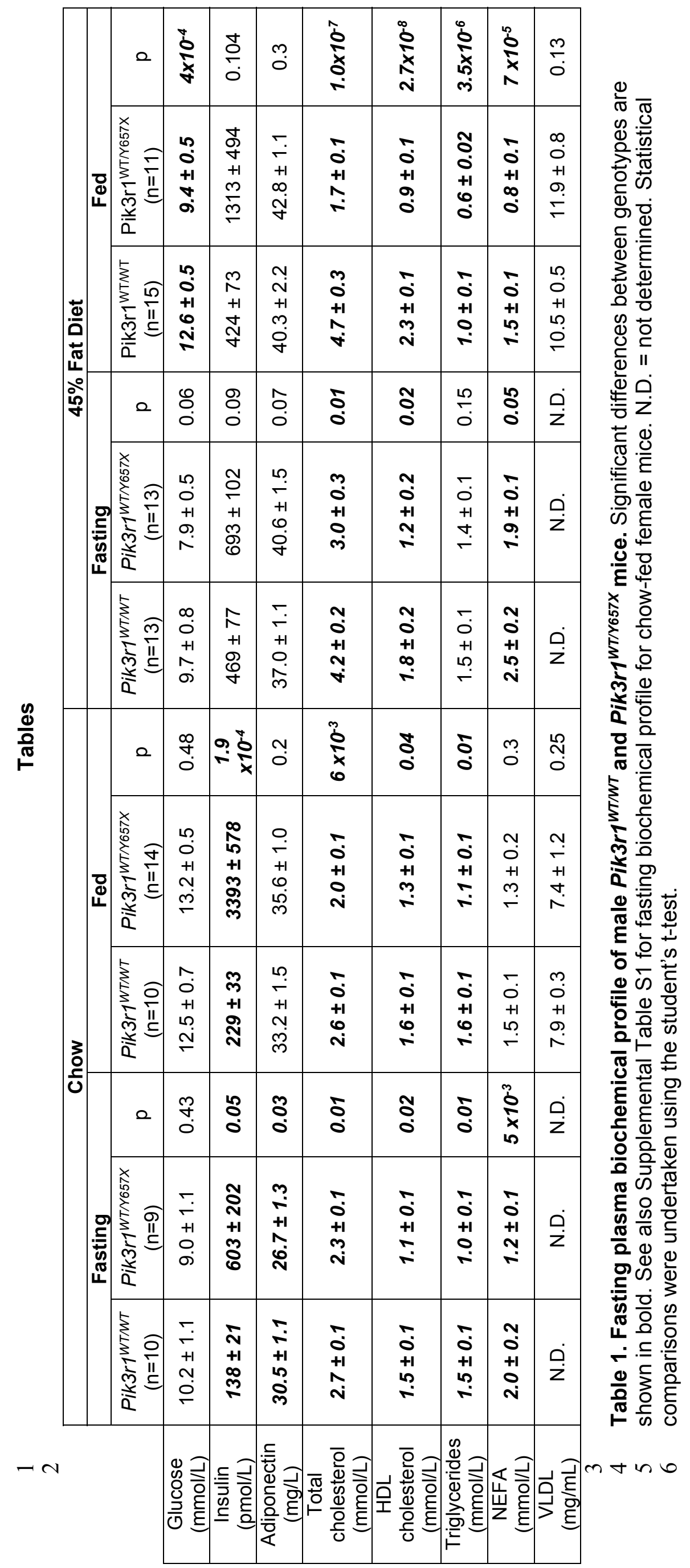




\section{Figure Legends}

1

2 Figure 1. Effect of Pik3r1 Y657X on prenatal development and postnatal growth

3 (A) Representative images and weights (adjacent scatter plot) of Pik3r1 ${ }^{W T / W T}$,

$4 \quad P i k 3 r 1^{W T / Y 657 X}$ and Pik3r1 $1^{Y 657 X / Y 657 X}$ embryos at E11.5. Scale bar $=5 \mathrm{~mm}$ (B)

5 Representative image of male $P i k 3 r 1^{W T / W T}$ and $P i k 3 r 1^{\text {WT/Y657X }}$ mice at 18 weeks old.

6 (C) Body lengths (nose to anus) at 18 weeks of Pik3r1 $1^{W T / W T}$ and Pik3r $1^{W T / Y 657 X}$ mice

$7 \quad(\mathrm{n}=11$ and 18 respectively) (D) Bodyweight increase from 8 to 16 weeks of

$8 P i k 3 r 1^{W T / W T}$ and $P i k 3 r 1^{W T / Y 657 X}$ (n=16 and 12 respectively). (E) Time course of fat

9 mass accretion, expressed as percentage of bodyweight, of $P i k 3 r 1^{W T / W T}$ and

$10 P i k 3 r 1^{W T / Y 657 X}$ mice $(\mathrm{n}=16$ and 12 respectively). Mean \pm S.E.M. are shown for

11 scatter plots in (A) and (C)-(E) (F)-(J) Masses of (F) inguinal adipose tissue

12 (IngWAT) $(\mathrm{G})$ Epididymal adipose tissue (eWAT), (H) Brown adipose tissue (BAT),

13 (I) Liver, and (J) Heart of Pik3r1 $1^{\text {WT/WT }}$ and Pik3r1 $1^{\text {WT/Y657X }}$ mice $(\mathrm{n}=11$ and 7

14 respectively). (K) Food intake ( $\mathrm{n}=13$ for $P i k 3 r 1^{W T / W T}$ and $\mathrm{n}=14$ for Pik3r ${ }^{\text {WT/Y657X) }}$ )

15 and (L) Energy expenditure ( $\mathrm{n}=7$ for $P i k 3 r 1^{W T / W T}$ and $\mathrm{n}=13$ for $P i k 3 r 1^{W T / Y 657 X}$ ) of wild

16 type and heterozygous mice assessed at 18 weeks old. All masses and energy

17 expenditure are shown relative to total lean mass, and were analysed statistically by

18 ANCOVA. $* * * *=p<0.0001$

21 Figure 2. Ovarian follicular appearance and profile of Pik3r1 $1^{\text {WT/Y657X }}$ mice and

22 wild type littermates Representative H\&E-stained cross sections of ovaries from 7

23 day old $P i k 3 r 1^{W T / W T}$ (A) and $P i k 3 r 1^{W T / Y 657 X}$ (B) mice. Scale bars $=300 \mu \mathrm{m}$. (C-G)

24 Examples of follicles at different stages of pre-antral follicular development: 
1 primordial $(C)$, transitional $(D)$, primary $(E)$, primary plus $(F)$ and secondary $(G) .(H)$

2 Cross sectional area of follicles from 7 day old Pik3r1 $1^{\text {WT/WT }}$ and Pik3r1 ${ }^{\text {WT/Y657X }}$ mouse

3 ovaries, classified by developmental stage ( $\mathrm{n}=3$ per genotype). (I) Proportions of

4 follicles at different developmental stages in Pik3r1 $1^{\text {WT/WT }}$ and Pik3r1 $1^{\text {WT/Y657X }}$ mouse 5 ovaries ( $\mathrm{n}=3$ per genotype). Bars represent mean \pm SEM.

9 Figure 3. Response of $P i k 3 r 1^{W T / Y 657 X}$ mice to a palatable $45 \%$ fat diet. (A)

10 Bodyweight increase from 8 to 16 weeks of Pik3r $1^{\text {WT/WT }}$ and Pik3r1 $1^{\text {WT/Y657X }}$ (n=16 and

1112 respectively). (B) Time course of fat mass accretion, expressed as percentage of 12 bodyweight, of $P i k 3 r 1^{W T / W T}$ and $P i k 3 r l^{W T / Y 657 X}$ mice ( $=16$ and 12 respectively).

13 Masses of (C) Epididymal adipose tissue (eWAT), (D) Inguinal adipose tissue 14 (IngWAT), (E) Brown adipose tissue (BAT), (F) Liver, and (G) Heart of Pik3r1 ${ }^{\text {WT/WT }}$ 15 and Pik3r1 $1^{W T / Y 657 X}$ mice ( $\mathrm{n}=12$ for both genotypes). (H) Representative histological 16 appearance of haematoxylin and eosin-stained eWAT from Pik3r1 ${ }^{W T / W T}$ and 17 Pik3r1 $1^{W T / Y 657 X}$ mice. Scale bars $=100 \mu \mathrm{m}$ (I) Adipocyte size distribution in eWAT 18 based on quantification of $>1,000$ cells per genotype from 4 wild type and 4 19 heterozygous mice. The inset shows a zoomed-in view of the early part of the 20 distribution. (J) Representative images of ex vivo differentiated stromovascular cells 21 from ingWAT stained with Oil Red O (see also Supplementary Figure S5). (K) Food 22 intake ( $\mathrm{n}=13$ for Pik3r1 $1^{W T / W T}$ and $\mathrm{n}=14$ for Pik3r1 $1^{W T / Y 657 X}$ ) and (L) Energy 23 expenditure ( $\mathrm{n}=17$ for $P i k 3 r 1^{W T / W T}$ and $\mathrm{n}=10$ for $\left.P i k 3 r 1^{W T / Y 657 X}\right)$ of wild type and 24 heterozygous mice assessed at 18 weeks old. All masses and energy expenditure are 25 shown relative to total lean mass, and were analysed statistically by ANCOVA. **** 
$1=\mathrm{p}<0.0001(\mathrm{M})$ Locomotor activity of Pik3r1 ${ }^{W T / W T}$ and Pik3r1 $1^{W T / Y 657 X}$ mice $(\mathrm{n}=17$

2 and $n-10$ respectively) Mean \pm SEM are shown. Statistical analysis used included 2-

3 way repeated measured ANOVA for (A-B), ANCOVA for (C-G, K and L), and

4 Student's t-test for $(\mathrm{M}) .{ }^{*} \mathrm{p}<0.05,{ }^{*} \mathrm{p}<0.01, * * * \mathrm{p}<0.001$ and $* * * * \mathrm{p}<0.0001$.

5

6 Figure 4. Pik3r1 $1^{W T / Y 657 X}$ mice show systemic and tissue-level insulin resistance (A)

7 Glucose infusion rates during hyperinsulinemic euglycemic clamping of Pik3r ${ }^{W T / W T}$

8 and $P i k 3 r 1^{W T / Y 657 X}$ mice on chow ( $\mathrm{n}=4$ and 4$)$ or $45 \%$ fat diet $(\mathrm{n}=10$ and 11$)$ at 16

9 weeks old; (B) Oral glucose tolerance test (OGTT) and corresponding comparison of 10 areas under the curves (AUC) of Pik3r1 $1^{W T / W T}$ and $P i k 3 r 1^{W T / Y 657 X}$ mice on chow at X

11 weeks old ( $\mathrm{n}=10$ and 9). (C) Insulin concentrations and AUC for the same OGTT.

12 (D) Insulin tolerance test and AUC comparison for the same mice 1 week later. (E)

13 Glucose disposal and (F) suppression of hepatic glucose output by insulin during 14 hyperinsulinemic euglycemic clamping of $P i k 3 r 1^{W T / W T}$ and $P i k 3 r 1^{W T / Y 657 X}$ mice on

15 chow at 18 weeks old (both $n=4)$. (G) Glycogen content of livers during a fasting16 refeeding cycle in chow fed animals at 16 weeks old (both $n=6)$. (H) Plasma non17 esterified free fatty acid concentrations during hyperinsulinemic euglycemic 18 clamping (both genotypes $n=4$ ). (I)-(L) Representative images of immunoblots and 19 corresponding quantifications of tissue lysates from mice injected intraperitoneally 20 with $2 \mathrm{U} / \mathrm{kg}$ insulin 10 mins prior to sacrifice, showing $\mathrm{pAkt}^{\mathrm{Ser} 473}$, total Akt and their 21 ratio: (I) Liver, (J) Skeletal muscle (K) eWAT, (L) ingWAT. (n=6 per genotype and condition). (M) Insulin-induced fold increase of glucose uptake into ex vivo incubated

23 soleus ( $\mathrm{n}=18$ for Pik3r1 $1^{W T / W T}, \mathrm{n}=11$ for $P i k 3 r 1^{W T / Y 657 X}$ ) and Extensor Digitorum

24 Longus (EDL) ( $\mathrm{n}=20$ and 11). (N) Representative immunoblots of Soleus and EDL

25 lysates from the same paradigm. (O) Quantification of pAkt ${ }^{\text {Ser473 }}$ to total Akt ratios 
1 from soleus and EDL immunblots ( $\mathrm{n}=5$ and 4 for both). Quantitative data are

2 presented as mean \pm SEM. Analysis used includes 2-way repeated measures

3 ANOVA (A-D, when 2-factors involved), Student's t-test for (B-H, M-O) and 1-way

4 ANOVA for (I-L). * ${ }^{*}=\mathrm{p}<0.05, * *=\mathrm{p}<0.01, * * *=\mathrm{p}<0.001$ and $* * * *=\mathrm{p}<$

50.0001.

6

7

8 Figure 5. Lipid handling and liver phenotype of Pik3r1 $1^{W T / Y 657 X}$ mice (A)

9 Representative images of Oil-Red-O-stained livers of chow-fed and 45\% fat diet-fed

$10 P i k 3 r 1^{W T / W T}$ and $P i k 3 r 1^{W T / Y 657 X}$ mice. Scale bars $=200 \mu \mathrm{m}(\mathrm{B})$ Hepatic triglyceride and

11 (C) Hepatic total cholesterol concentration during a fasting refeeding cycle of chow

12 fed mice at 16 weeks old ( $\mathrm{n}=6$ per genotype). (D) Lipid tolerance testing and

13 comparison of areas under the curve (AUC), also of chow-fed mice, at 16 weeks old.

14 Triglyceride concentrations were equalised at baseline by adding the difference

15 between genotypes to the lower (heterozygous value), and the same fixed correction

16 was applied to all points on the graph $(n=11$ and $n=17$ for wild-type and

17 heterozygous mice respectively) (E) Faecal energy content determined by bomb

18 calorimetry of chow fed mice at 16 weeks old ( $\mathrm{n}=8$ and 8$)$. (F)-(L) Liver mRNA

19 expression, determined by quantitative real time PCR of (F) Srebpl and its

20 transcriptional targets (G) Acc1, (H) Fasn, and (I) Scd1, and of (J) Srebp2 and its

21 transcriptional targets (K) $M v k$ and (L) $N s d h l$ in chow fed mice during a fasting

22 refeeding cycle at 16 weeks old ( $\mathrm{n}=6$ per genotype per condition). Numerical data

23 are presented as mean \pm SEM, and were analysed by Student's t-test and 2-way

24 repeated measures ANOVA when appropriate. $*=\mathrm{p}<0.05$. 
bioRxiv preprint doi: https://doi.org/10.1101/224485; this version posted November 24, 2017. The copyright holder for this preprint (which

was not certified by peer review) is the author/funder, who has granted bioRxiv a license to display the preprint in perpetuity. It is made available under aCC-BY 4.0 International license.

Pik3r1WT/WT

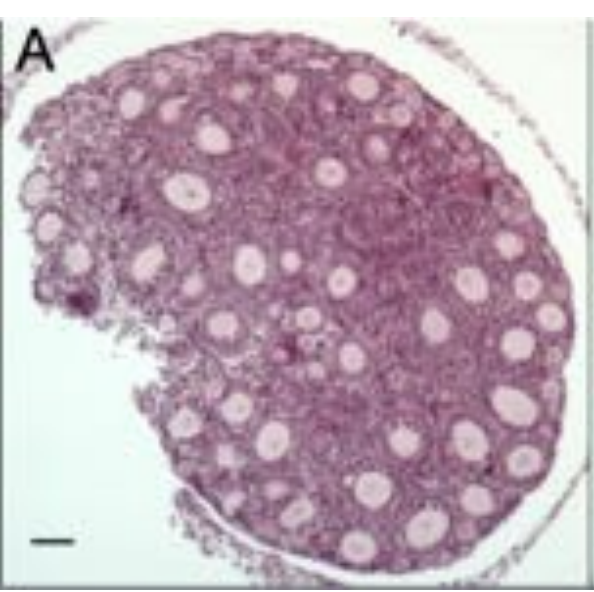

Pik3r1WT/Y657X

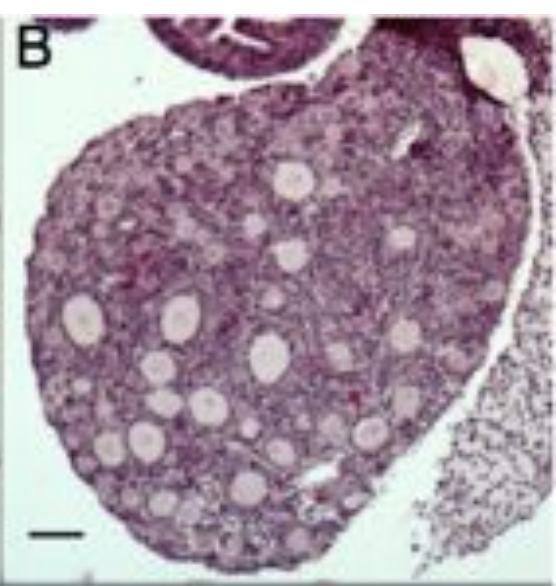

8

$\mathrm{H}$

हू

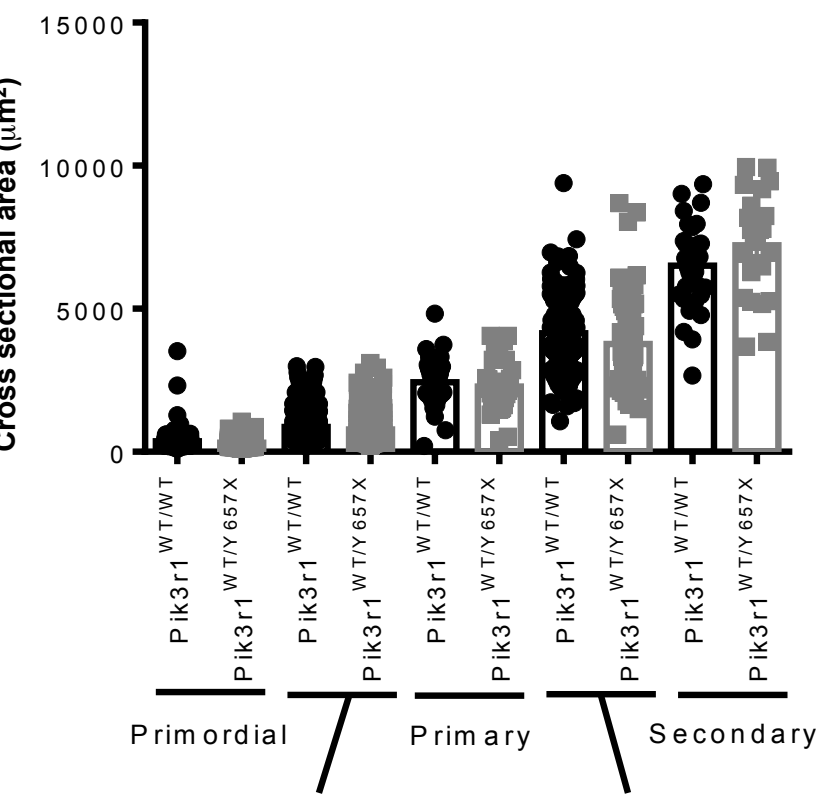

Transitional

Prim ary plus

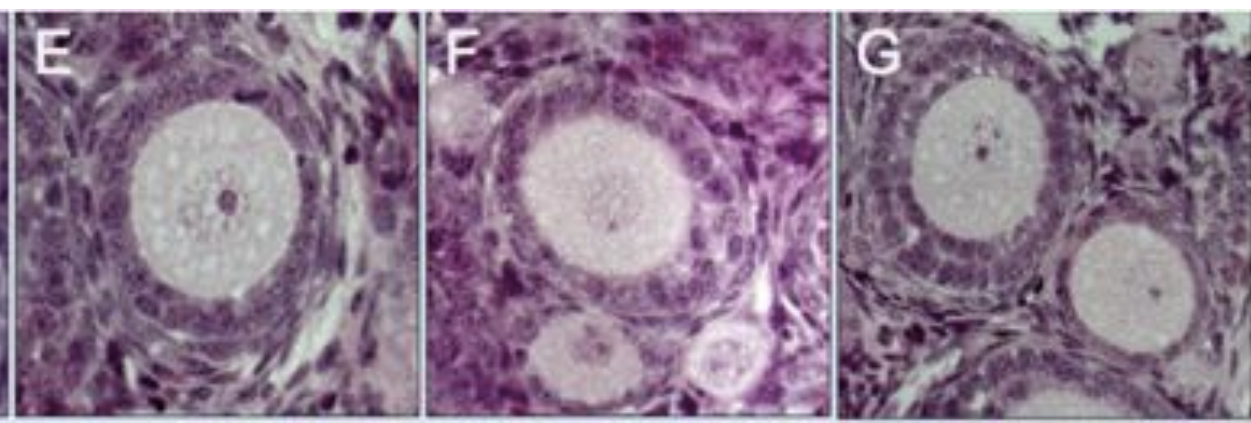

I

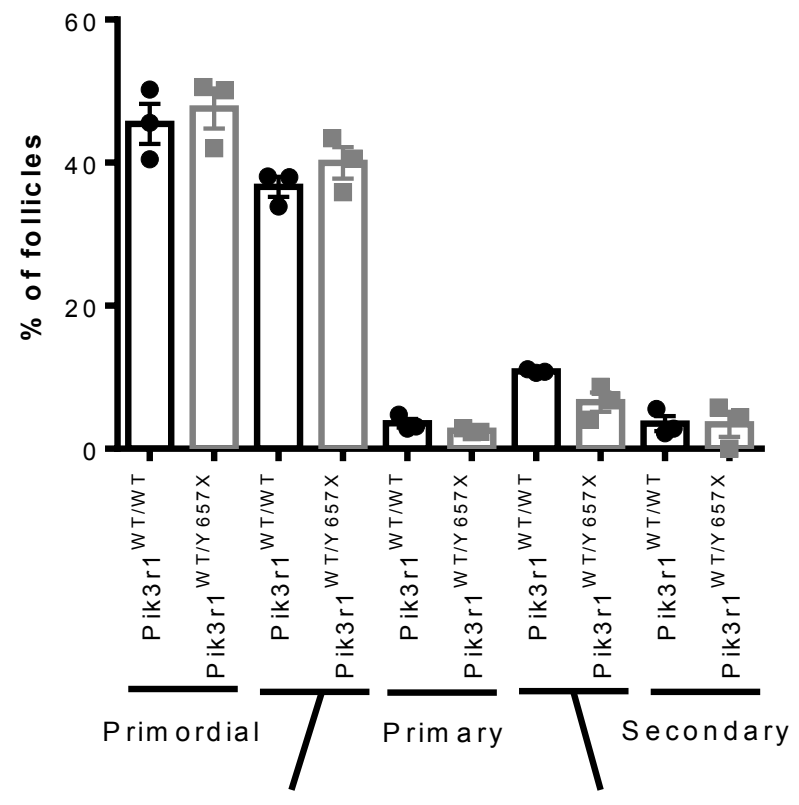

Transitional

Primary plus

Fig. 2 
